\title{
вмJ Global Health Community health workers for pandemic response: a rapid evidence synthesis
}

\author{
Soumyadeep Bhaumik (10 , ${ }^{1,2}$ Sandeep Moola, ${ }^{1}$ Jyoti Tyagi, ${ }^{1}$ Devaki Nambiar, ${ }^{1,2,3}$ \\ Misimi Kakoti ${ }^{1}$
}

To cite: Bhaumik S, Moola S, Tyagi J, et al. Community health workers for pandemic response: a rapid evidence synthesis. BMJ Global Health 2020;5:e002769. doi:10.1136/ bmjgh-2020-002769

- Additional material is published online only. To view please visit the journal online (http://dx.doi.org/10.1136/ bmjgh-2020-002769).

Received 29 April 2020 Revised 13 May 2020 Accepted 14 May 2020
Check for updates

(c) Author(s) (or their employer(s)) 2020. Re-use permitted under CC BY. Published by BMJ.

${ }^{1}$ The George Institute for Global Health, Vishakhapatnam, India ${ }^{2}$ The George Institute for Global Health, Faculty of Medicine, University of New South Wales, Sydney, New South Wales, Australia

${ }^{3}$ Prasanna School of Public Health, Manipal Academy of Higher Education, Manipal, India

\section{Correspondence to} Dr Soumyadeep Bhaumik; sbhaumik@georgeinstitute. org.in

\section{ABSTRACT}

Introduction Coronavirus disease (COVID-19), affects 213 countries or territories globally. We received a request from National Health Systems Resource Centre, a public agency in India, to conduct rapid evidence synthesis (RES) on community health workers (CHWs) for COVID-19 prevention and control in 3 days.

Methods We searched PubMed, websites of ministries $(n=3)$, public agencies $(n=6)$, multilateral institutions $(n=3)$, COVID-19 resource aggregators $(n=5)$ and preprints $(\mathrm{n}=1)$ (without language restrictions) for articles on CHWs in pandemics. Two reviewers screened the records independently with a third reviewer resolving disagreements. One reviewer extracted data with another reviewer cross-checking it. A framework on $\mathrm{CHW}$ performance in primary healthcare not specific to pandemic was used to guide data extraction and narrative analysis.

Results We retrieved 211 records and finally included 36 articles. Most of the evidence was from low-and middle-income countries with well-established CHW programmes. Evidence from $\mathrm{CHW}$ programmes initiated during pandemics and for $\mathrm{CHW}$ involvement in pandemic response in high-income countries was scant. CHW roles and tasks change substantially during pandemics. Clear guidance, training for changed roles and definition of what constitutes essential activities (ie, those that must to be sustained) is required. Most common additional activities during pandemics were community awareness, engagement and sensitisation (including for countering stigma) and contact tracing. CHWs were reported to be involved in all aspects of contact tracing - this was reported to affect routine service delivery. CHWs have often been stigmatised or been socially ostracised during pandemics. Providing PPE, housing allowance, equal training opportunities, transportation allowance, improving salaries (paid on time and for a broad range of services) and awards in high-profile public events contributed to better recruitment and retention. We also created inventories of resources with guiding notes on guidelines for health workers $(n=24)$, self-isolation in the community $(n=10)$ and information, education and counselling materials on COVID-19 $(n=16)$.

Conclusions CHWs play a critical role in pandemics. It is important to ensure role clarity, training, supportive supervision, as well as their work satisfaction, health and well-being. More implementation research on $\mathrm{CHWs}$ in pandemics is required.
Key questions

What is already known?

- Many countries with existing community health worker (CHW) programmes are mobilising CHWs to assist with COVID-19 response, while some are considering initiating new $\mathrm{CHW}$ programmes.

What are the new findings?

- In previous pandemics, CHWs have played several roles including for generating community awareness countering stigma and contact tracing. CHW engagement in contact tracing might hamper routine primary service delivery. Policies, guidance and training for these had to be developed.

- Disruption in supply chain, logistics and supportive supervision for CHWs have been common in pandemic scenarios. CHWs have been at increased risk for contracting disease.

- In the course of pandemics, CHWs have experienced stigmatisation, isolation and socially ostracisation. Improved remuneration, additional incentives, public recognition countering societal stigma, provision of psychosocial support and personal protective equipment were reported to be enablers.

\section{What do the new findings imply?}

- Roles of CHWs in a pandemic context need to be clearly defined with provision of adequate guidance, training and support. Contact listing and identification should be ideally be done by separate trained cadre while CHWs focus on essential service delivery. Ensuring the health, safety, well-being and support for CHWs is essential.

- There is not much evidence about initialisation of new CHW programmes during pandemics. Considering the complexity of barriers faced even in contexts with well-integrated programmes, ambitious programmes need to be considered with caution.

- There is a need for more research on CHWs in pandemics like COVID-19, especially with a focus on equity, gender and economic evaluation.

\section{INTRODUCTION}

The COVID-19 outbreak that originated in Wuhan City, China, in December 2019 now 
affects 213 countries or territories across the globe. Better health workforce utilisation and support are key pillars to enhance health systems capacity. ${ }^{12}$ Community health workers (CHWs) constitute a significant proportion of frontline health workforce in many countries ${ }^{3}$ and can potentially play an important role in control and prevention of pandemics like COVID-19.

We received a request to examine the evidence to inform the potential role, enablers and barriers for CHWs during COVID-19 prevention and control from the National Health Systems Resource Centre (NHSRC), India. The NHSRC is a public agency with the mandate to provide technical assistance to India's National Health Mission, a flagship health systems reform exercise initiated in 2005. An inventory of resources that government decision makers and technical agencies could rapidly scan to help develop guidelines, standards of procedures, advisory notes and communication materials was also deemed to be a useful addendum by the requesters. We conducted a rapid synthesis of global evidence that aimed to:

- Understand key roles, issues, barriers and enablers for CHWs for pandemic response.

- Develop an inventory of resources that could be used to develop guidance, training manuals and information, education and communication (IEC) materials related to COVID-19 for CHWs.

Rapid evidence synthesis (RES) is an emerging arena within the domain of evidence synthesis wherein a balance is struck between accuracy and timelines in the conduct of evidence synthesis to inform health policy and systems decision making. ${ }^{4}$

\section{METHODS}

\section{Approach for the study}

Considering the urgency of evidence to inform decision making, we were provided a three-calendar day deadline by the requester. Based on an initial scoping, discussion with the requesters and the emergent nature of COVID19, we expanded the scope to understand what can be learnt from recent pandemics, that is, severe acute respiratory syndrome (SARS), swine flue, Ebola virus disease (EVD) and Middle East respiratory syndrome coronavirus (MERS-CoV). We followed an RES approach, wherein processes and methods of the traditional systematic review approach were tailored to ensure timeliness. ${ }^{4}$ We took a broad scoping review approach for the RES to support the wide nature of decisions under consideration. While the request form NHSRC was in the context of government-run CHW programmes in India (Accredited Social Health Activists or ASHAs and Aganwadi workers), the scope of the synthesis was not restricted based on governance, financing or scale of the programme, owing to the need to capture the greatest range of relevant evidence in pandemic contexts. There are no reporting guidelines specifically for RES, so we used the Preferred Reporting Items for Systematic Reviews and
Meta-Analyses (PRISMA) checklist for scoping reviews. ${ }^{5}$ The PRISMA checklist is presented in online supplementary appendix 1 .

\section{Eligibility criteria}

We included studies that met the following criteria:

- Concept and context: studies or guidance related to roles, issues, challenges and enablers of CHWs during any phase of recent pandemics. The pandemics considered were SARS, swine flu, EVD, MERS-CoV and COVID-19.

- Types of participants: we did not use any specific definition of CHW recognising the fact that there are identified differently in different health systems across the world. We included studies with CHWs as participants (as defined by primary study authors). Synonyms for CHWs including but not limited to the following were also included:

- Community health aide/practitioner/provider.

- Lay health worker/aide/practitioner/provider/ advisor/promoter.

- Frontline health worker or community worker.

- Rural/village/outreach health worker.

- Community volunteer/worker/surveillance volunteer.

- Multipurpose health worker or health extension worker or health auxiliary.

- Types of evidence sources: We included following types of evidence sources: - primary research studies (of any design-qualitative or quantitative), any evidence synthesis, guidelines, training materials, advisories, standards of procedures and technical reports.

There was no language, date or geographical restrictions.

\section{Search methods for identification of records}

We searched PubMed given the need to complete the RES in 3 days (search strategy in online supplementary appendix 2). We screened the reference list of all included studies to identify additional records and hand searched (MK and JT) 18 websites of different government ministries (India, Australia and Singapore), public health agencies (from China, US, South Africa, UK, Hong Kong and Australia), multinational agencies (WHO, European CDC and African CDC), COVID-19 resource aggregators available at the time of review (Wiley, Elsevier, Oxford University Press, New England Journal of Medicine, Journal of the American Medical Association) and preprints (medRxiv) (online supplementary appendix 3).

\section{Screening, data collection and analysis}

Two reviewers independently screened the titles and abstracts of studies for inclusion with a third author resolving consensus ( $\mathrm{SB}, \mathrm{SM}$ and $\mathrm{JT}$ ). At the full-text level, decision for inclusion or exclusion was done with the consensus of three authors (SB, SM and JT). Data from included studies were extracted using a predefined 
template by one reviewer (SM, JT, SB and DN) and crossverified by at least one other reviewer. Cross-verification was not possible for the two French studies as only one of the reviewers knew the language (DN). No assessment of methodological quality of the studies was conducted as a part of methodological tailoring to expedite review process. ${ }^{4}$

We adapted an existing conceptual framework for assessment of CHW performance in primary healthcare ${ }^{6}$ to guide data extraction and narrative synthesis. The framework looks at inputs, programmatic processes, CHW-level and community-level outputs to improve health outcomes in the context of economic evaluation and equity, gender and accountability. The framework is not specific to pandemic response; for ease of reading and clarity, some components of the framework were merged, and issues related to multiple aspects of the framework were mentioned without repetition.

\section{Patient and public involvement}

The RES did not include patients, CHWs or the public in its conduct. The RES has been disseminated to relevant stakeholders and the public as noted in the subsequent section on policy impact and stakeholder engagement.

\section{RESULTS}

\section{Search results and study selection}

We searched PubMed on 21 March 2020 and retrieved 211 records. Following title and abstract screening, fulltext articles were retrieved for 36 potentially relevant

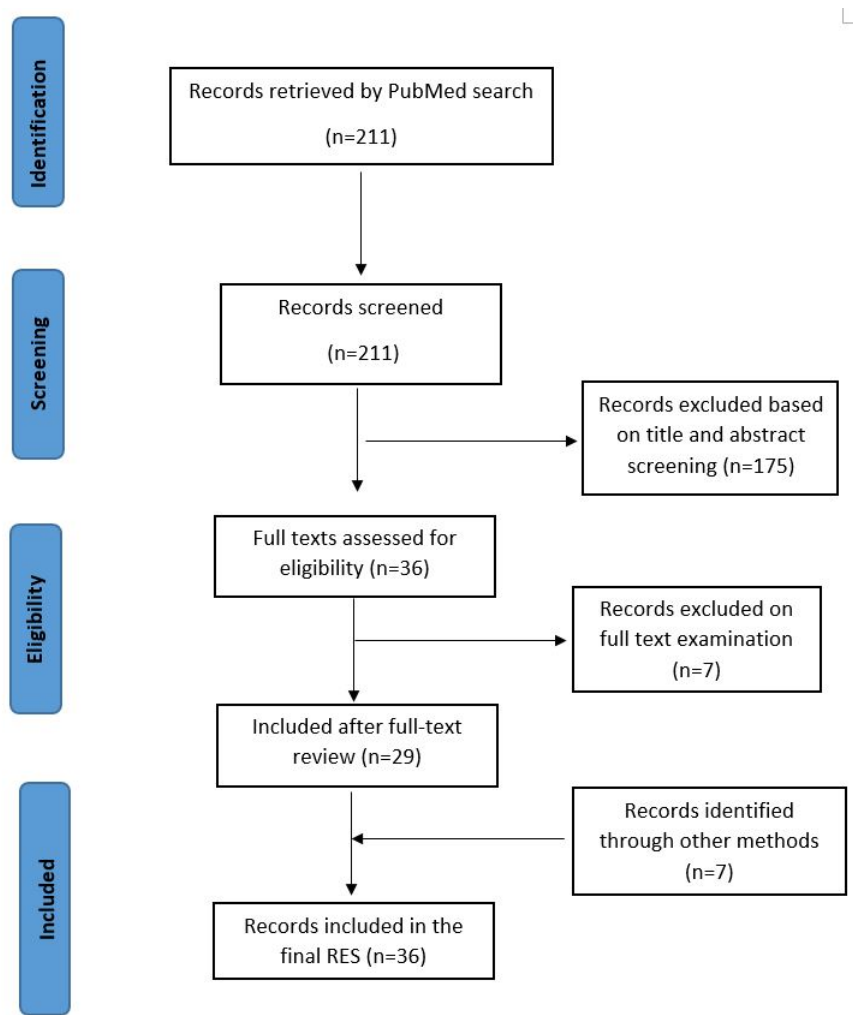

Figure 1 PRISMA flow chart for included studies. PRISMA, Preferred Reporting Items for Systematic Reviews and MetaAnalyses. studies. Seven records were excluded following full-text examination, leaving 29 studies for inclusion. An additional seven studies were identified by screening reference lists of the initial 29 studies that were included. Overall, 36 studies were included in the rapid review. A PRISMA flow chart for the same is presented in figure 1 . A list of studies excluded at full-text level is presented in online supplementary appendix 4.

\section{Characteristics of included studies}

A majority of the studies included in the review were qualitative $(n=11)^{7-17}$ followed by descriptive crosssectional studies $(n=9),{ }^{18-26}$ pretest and post-test studies $(\mathrm{n}=6),{ }^{27-32}$ literature reviews $(\mathrm{n}=3),{ }^{33-35}$ mixed-methods studies $(\mathrm{n}=2),{ }^{36}{ }^{37}$ guidelines $(\mathrm{n}=2),{ }^{38}{ }^{39}$ one systematic review, ${ }^{40}$ one randomised controlled trial $^{41}$ and one case report. $^{42}$

Key characteristics of the included studies are presented in table 1 . Studies were conducted in lowincome and middle-income countries or LMICs (Benin, Burundi, Cambodia, Democratic Republic of Congo, Ghana, Guinea, Kenya, Liberia, Mali, Nepal, Nigeria, Sierra Leone, Tanzania, Uganda, Vietnam, Zambia and Zimbabwe) and also in high-income countries (Iceland, UK, USA). The two guidelines were published by the WHO. The three literature reviews and the systematic review had included studies from multiple LMICs in Southeast Asia, South America and Africa.

Reporting for context of CHWs programmes in the studies was not consistent. Notwithstanding this, the bulk of the evidence from LMICs is from countries that had existing government managed CHW programmes (government or aid funded) with a few exceptions. These exceptions were an existing government-funded programme in Nigeria managed by a non-government faith-based organisation and a new government CHW program being initiated in Vietnam. ${ }^{7}$ In addition, another study looked at pandemic related training being provided by non-government agencies to existing CHWs in Nigeria. ${ }^{30}$ CHWs in LMICs are volunteers with no permanent base-pays and are paid small amounts (in cash or kind) on the basis of actual service delivery. The high-income country evidence pertained to pandemic preparedness in Iceland ${ }^{9}$; USA, wherein a new CHW program was initiated during a pandemic wherein CHWs were integrated as staff; and the UK where potential availability of volunteers for CHWs was studied. ${ }^{1441}$ As such, the evidence from high-income countries is very limited, and unless otherwise stated, evidence in subsequent section pertains to LMIC contexts.

\section{Summary of findings on role, issues, enablers and barriers for CHWs in pandemic response}

The included studies examined the role of CHWs in disease outbreak situations, in addition to exploring the barriers and enablers of working in pandemics. Overall, the studies reported that CHWs had a role to play in terms of community engagement (mostly in rural areas) 


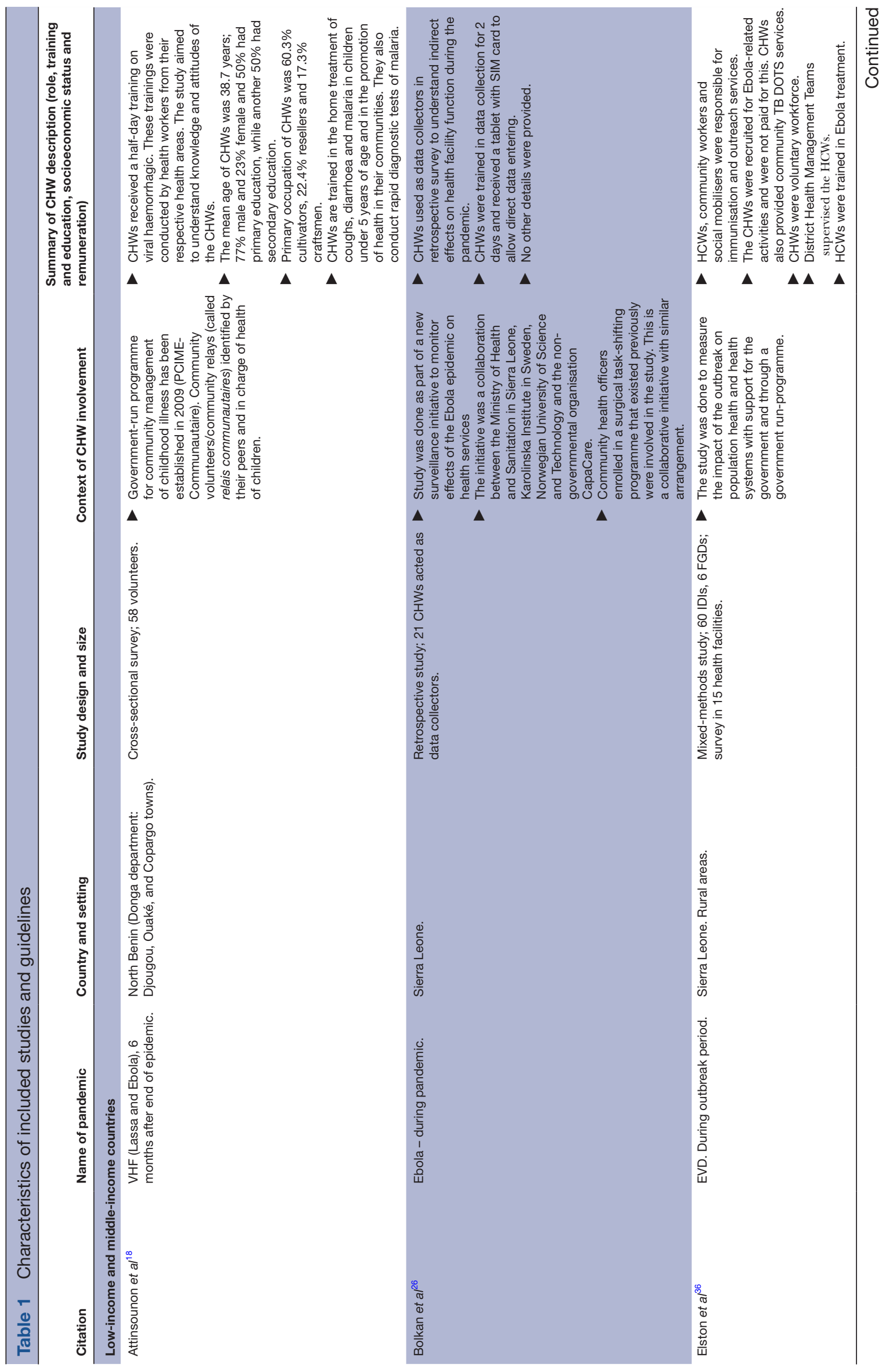

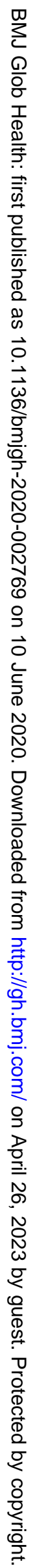




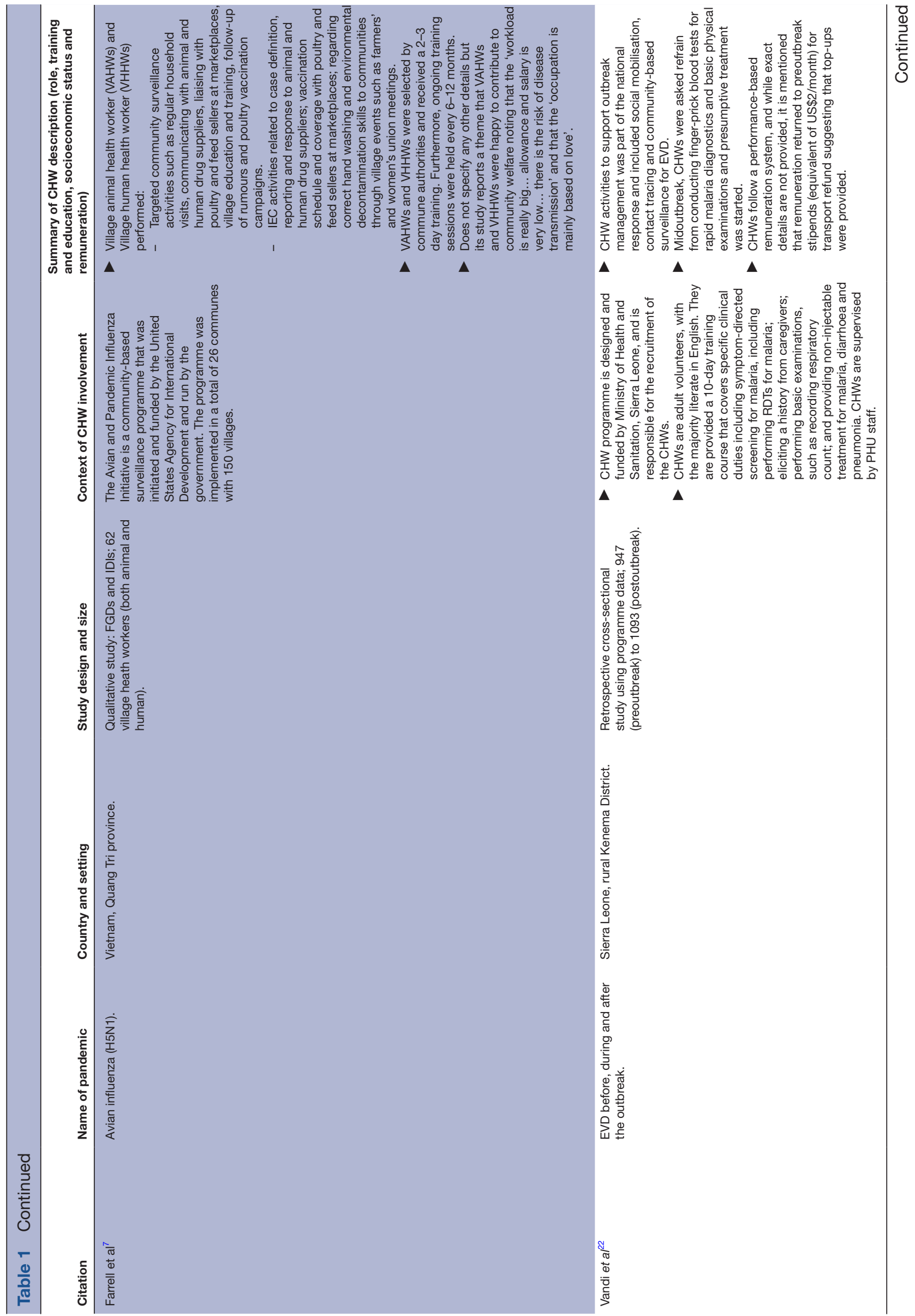




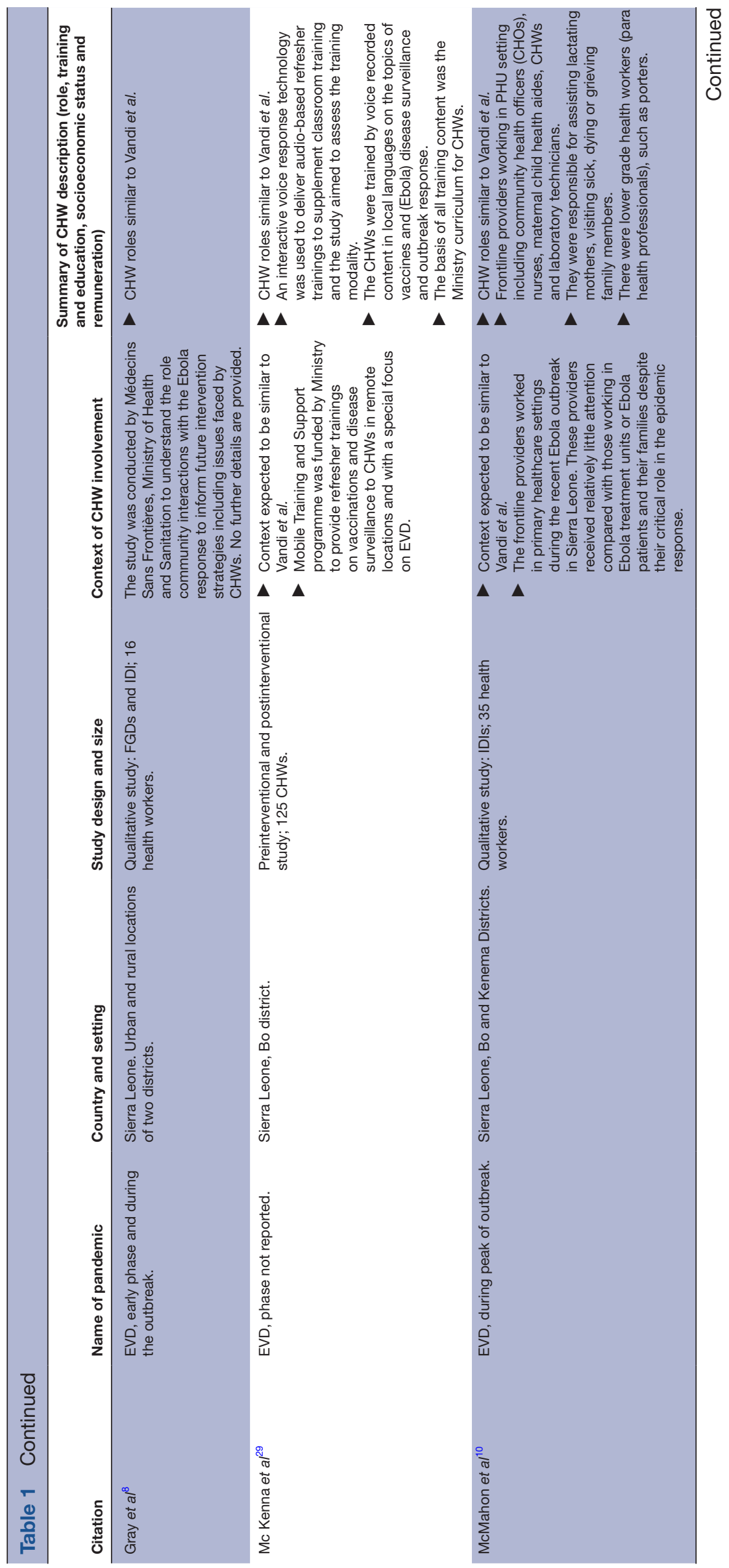




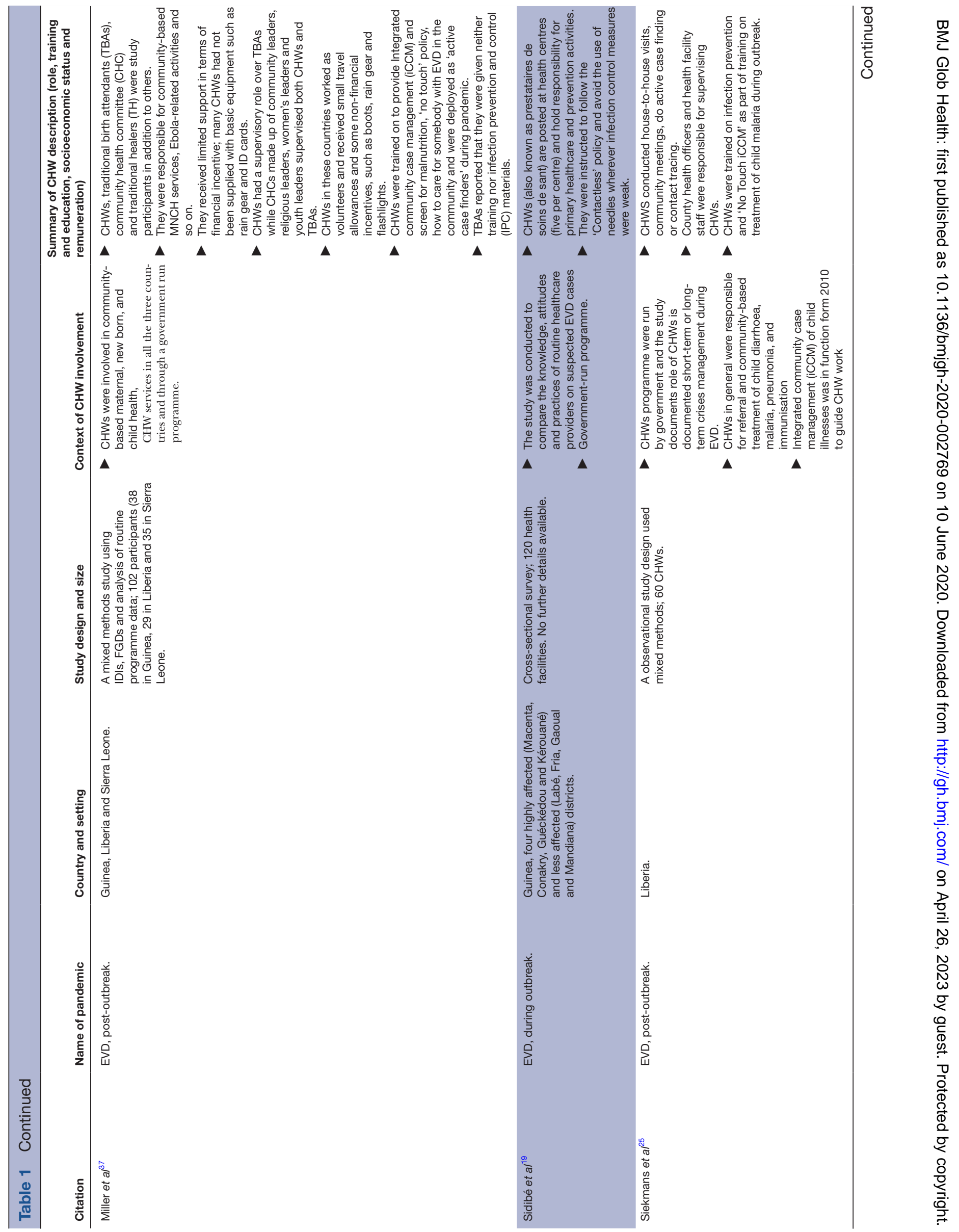




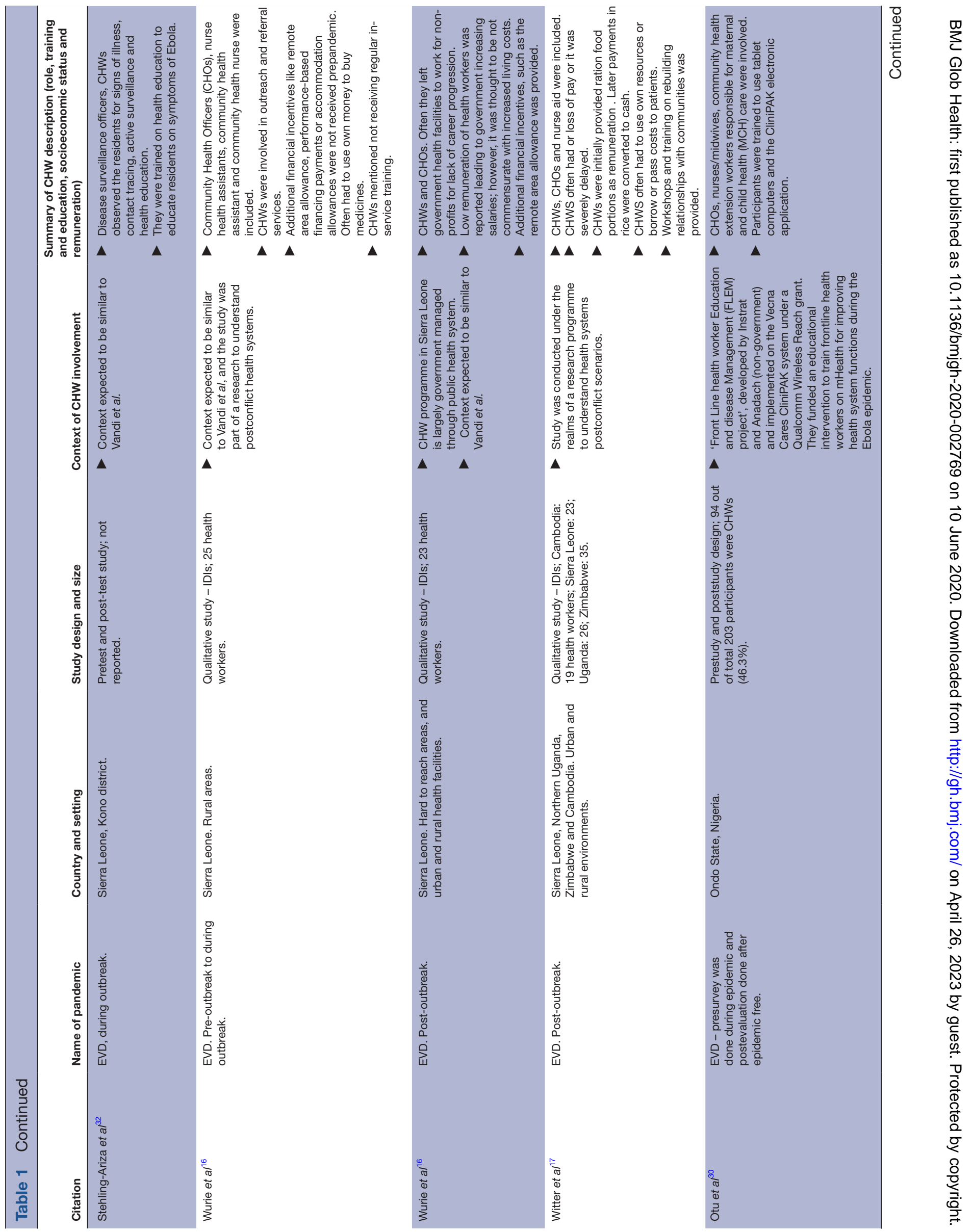




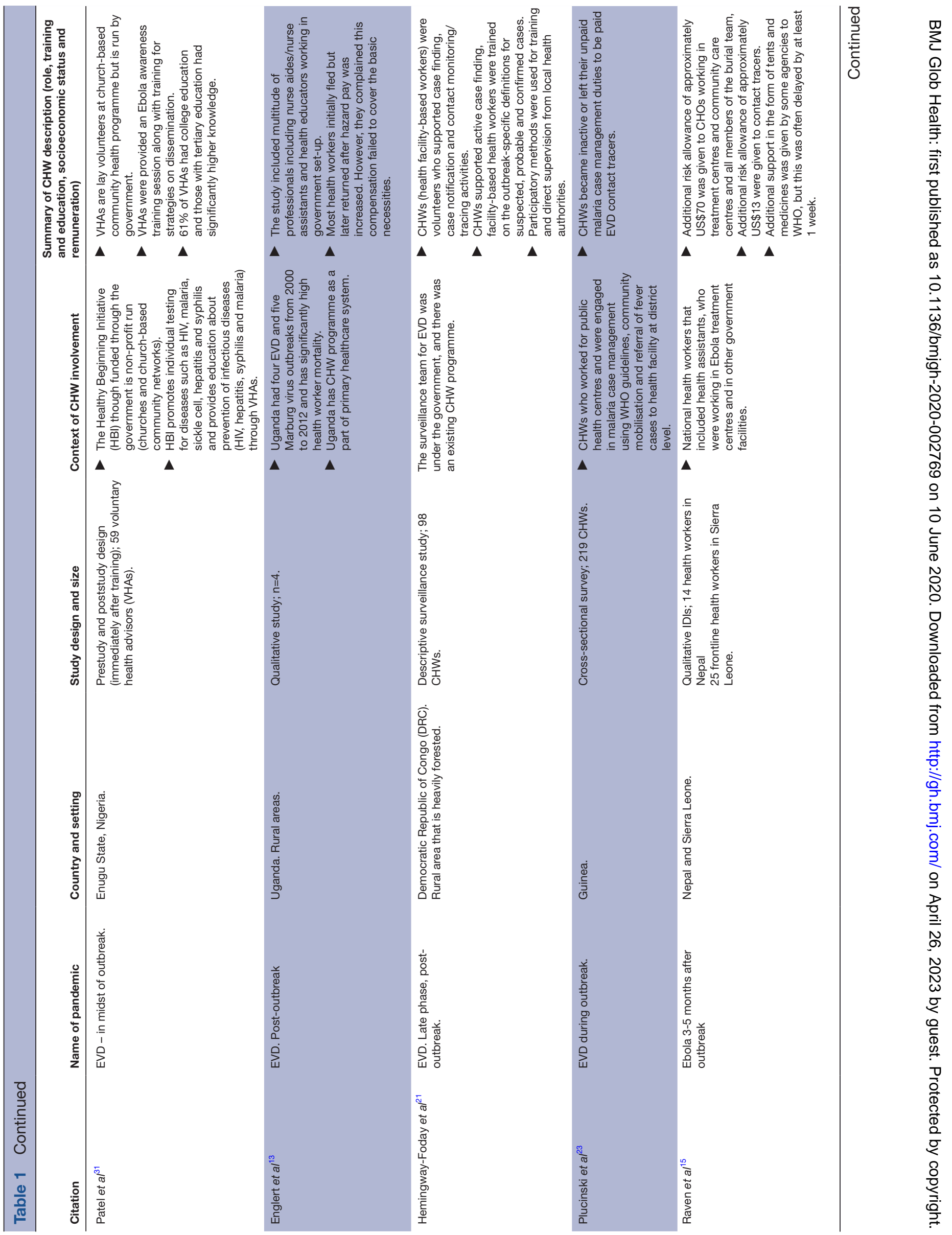



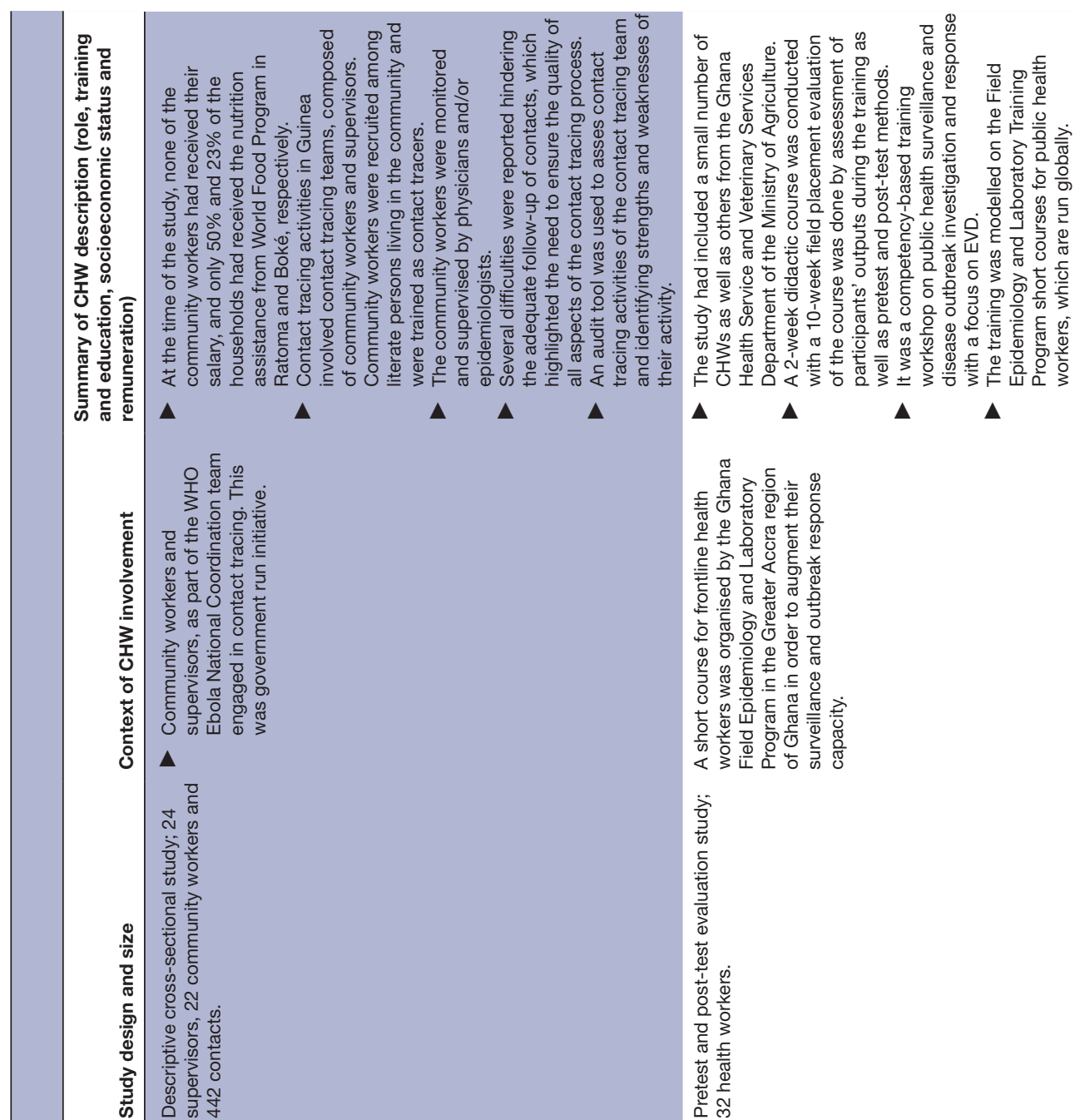

당

政

管产产

वृ 를

क

응

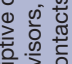

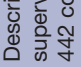

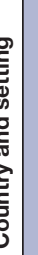

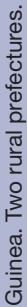

竞

ब

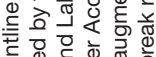

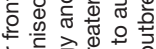

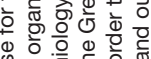

究

훙

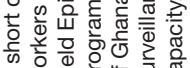

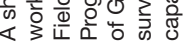

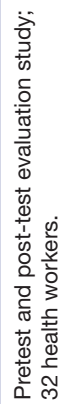

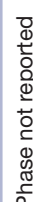

学 $\frac{\mathbb{\Phi}}{0}$

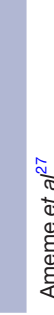

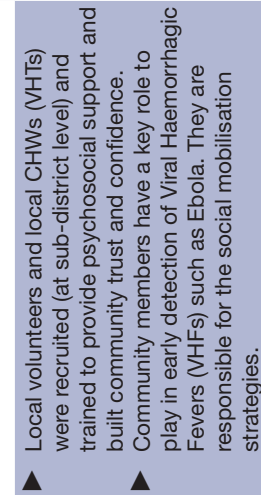

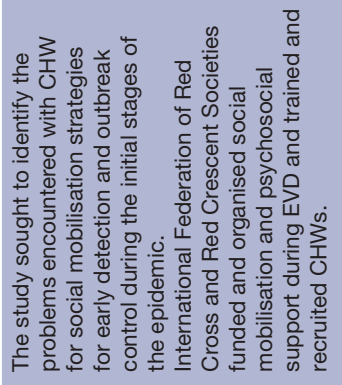

$\Delta$

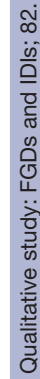

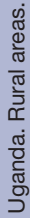

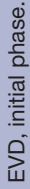

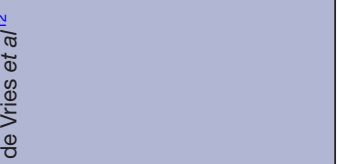

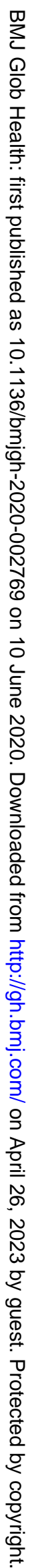




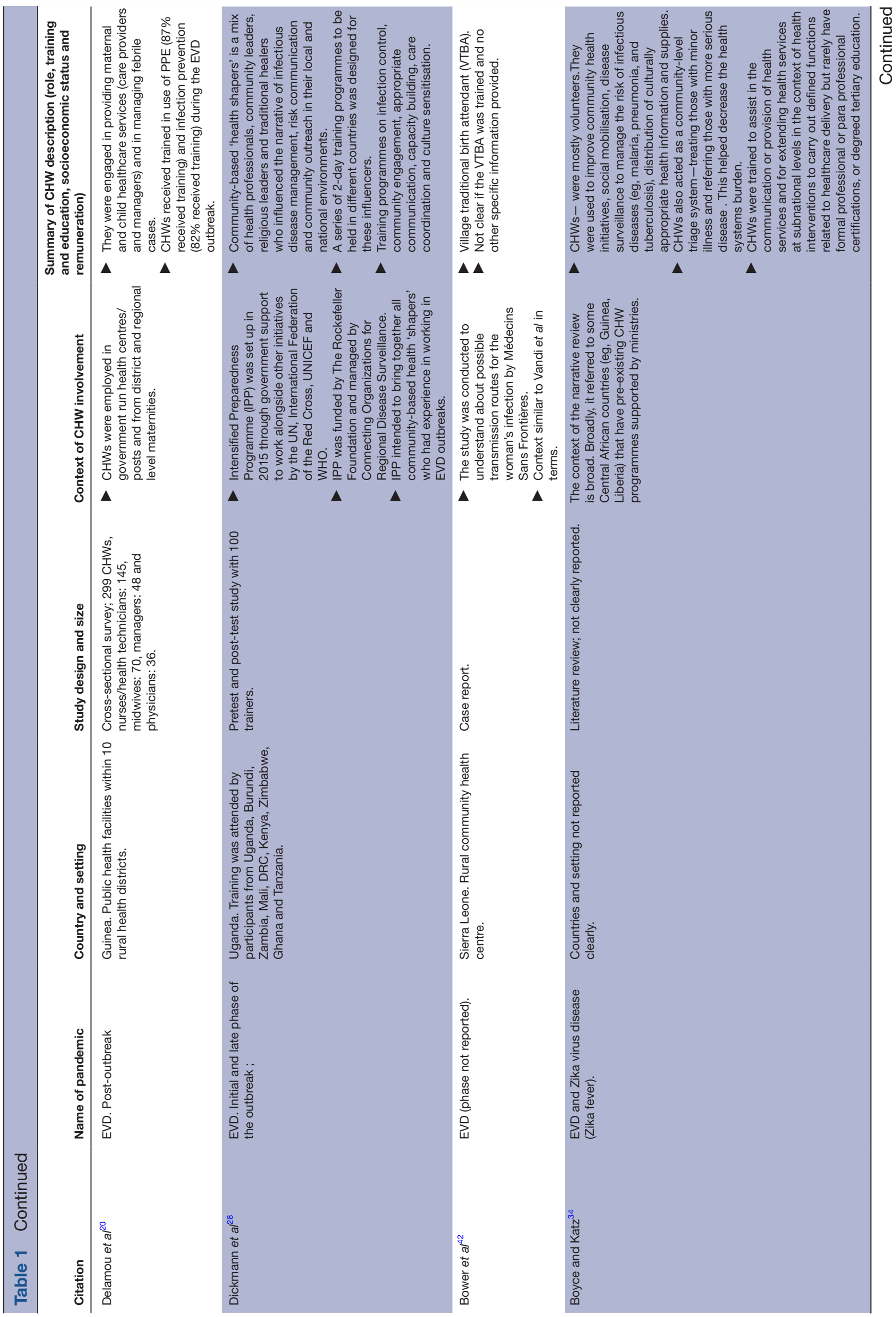

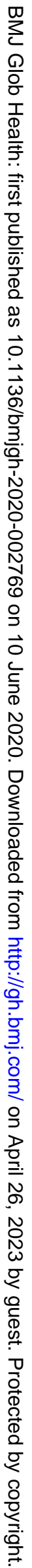




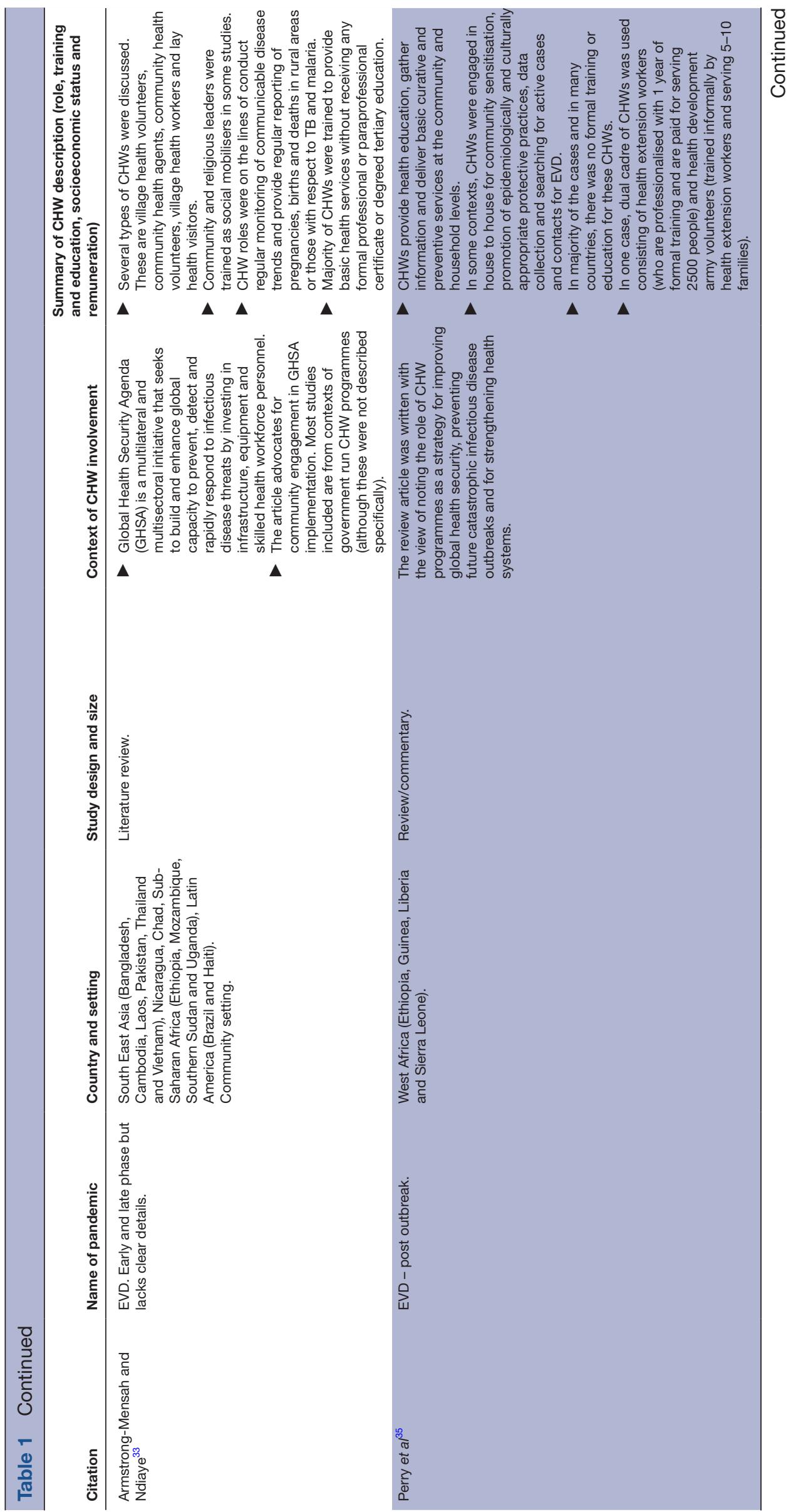

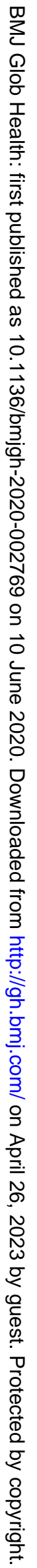




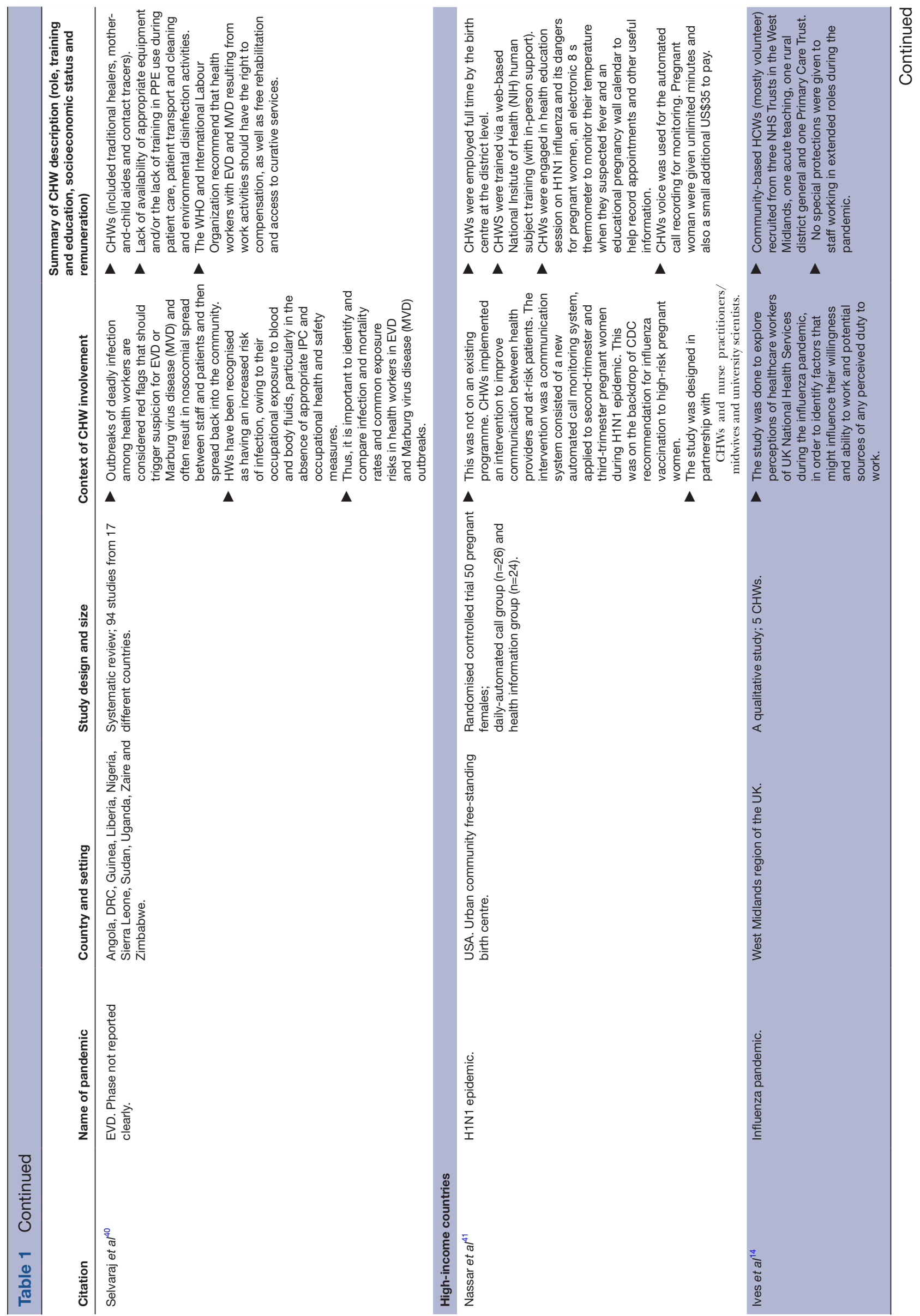




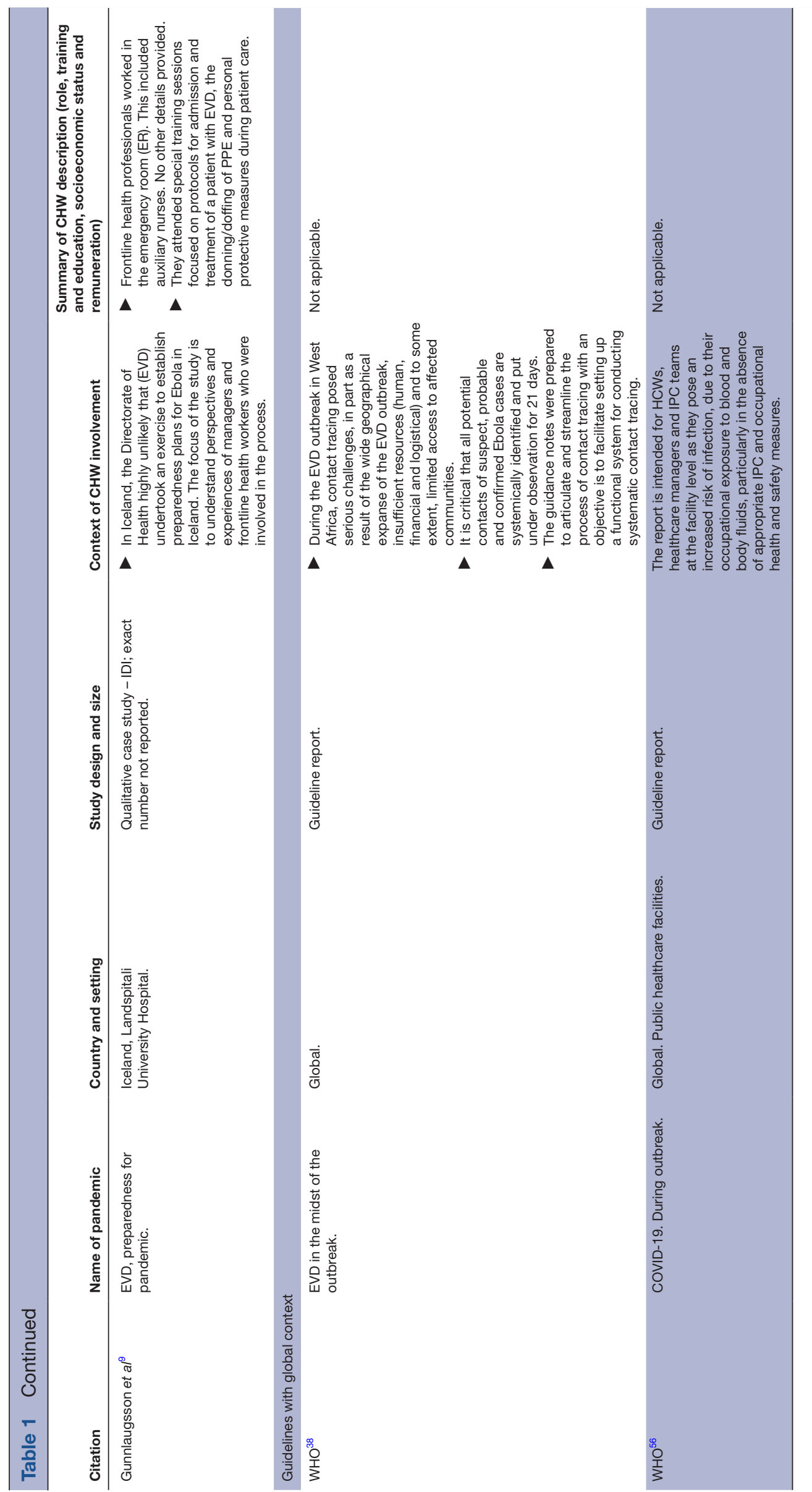

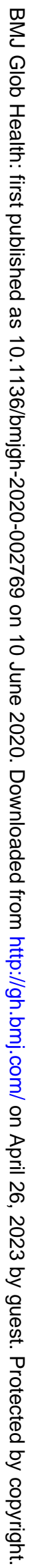


and creating awareness during pandemic responses. The key findings from the included studies are presented as per the elements of the conceptual framework. ${ }^{6}$

\section{Input in CHW programmes during pandemic responses}

Myriad inputs and planning were required to ensure effective pandemic response through CHWs. These are summarised in subdomains below:

- Policies related to CHW task/roles. Policies and guidance related to specific roles and tasks for CHWs were reported in many studies. ${ }^{9} 2130343738$ These policies largely aimed to distinguish tasks as essential (routine activities that need to be continued but with modifications for decreased transmission risk), non-essential (non-essential activities that could be postponed) and additional activities that need to be carried. New roles and tasks for CHWS pertaining specifically to pandemic control varied immensely. This included community awareness (related to disease risk factors and symptoms, appropriate preventive practices and countering stigma), engage-

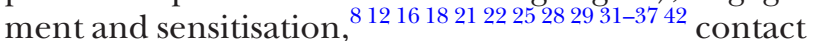
tracing, ${ }^{21} 22242532$ visiting sick or grieving community members, ${ }^{10}$ targeted community surveillance for prevention using one-health approaches ${ }^{7}$ and data collection to understand the impact of a pandemic ${ }^{26}$ in LMICs. In USA, they were engaged for better risk communication to high-risk groups. ${ }^{41}$ The other two studies from high-income countries did not indicate specific CHW roles.

The WHO's guidance on contact tracing recommends contact identification and listing to be conducted by a trained epidemiologist or surveillance officer, while contact follow-up might be done through CHWs. ${ }^{38}$ However, polices to involve CHWs in aspects of contract tracing had been developed and implemented during EVD, but their appropriateness is not well understood. ${ }^{21222425}$ It has further been noted that CHWs had completely abandoned their routine unpaid malaria case management duties over preferences to the paid contact tracing work. ${ }^{23}$ Changes in policies and standards of procedures for routine service delivery (eg, 'no-touch' policies for community level screening and treatment) were also reported. ${ }^{25} 3237$ Policies for defining CHW roles were reported to be crucial for the purpose of bringing accountability and building trust in health systems, although this was not discussed more specifically.

- Logistics and funding for CHW programmes. Disruptions in drug and equipment supplies were common during pandemics and policies and mechanisms to address them from an early phase of the outbreak were reported to be important. ${ }^{5} 10{ }^{16}$ In addition, shortfalls in routine supportive supervision methods may be expected. Sustained investments in CHWs were required during pandemic response including revisiting financial investments (discussed subsequently) for optimal outcomes. ${ }^{34}$ Issues in relation to financial incentives for CHWs are further discussed in section on CHW motivation and well-being.

- Governance and stakeholders. A wide range of stakeholders in the community needed to be engaged. The evidence base on stakeholder engagement is mostly from LMICs with existing CHW programmes where they were additionally engaged for COVID-19 activities and has been discussed in a subsequent domain on community support.

- Information management systems for CHWs in pandemics. Operational support using information management systems and digital health technology were reportedly used for supporting and monitoring CHW programmes. Honesty, transparency of communication and reciprocity of information with CHWs were noted to be beneficial. ${ }^{9} 1421$ Many studies reported that pandemics impacted supervision and support for CHWs and health management information systems. Disease surveillance systems (reliant on passive reporting from health facilities) became almost entirely non-functional in some cases. ${ }^{36}{ }^{43} \mathrm{In}$ Vietnam, a new community based surveillance component was developed, in which CHWs played a critical role. ${ }^{22} \mathrm{CHW}$ recorded their daily activities in registers, and their reports were collated by the peripheral health unit supervisor and entered electronically, which formed the basis of information reaching the Ministry. The requirement to travel long distance to attend supervision meetings, health worker shortage, facility closures, restrictions on movement and increased prioritisation pandemic work were identified as key challenges for routine supervision and health information systems management. ${ }^{25} 37$

Programmatic process and health systems were crucial for effective service delivery by CHWs during pandemics.

Supportive systems, development, competency and well-being for CHWs

CHWs and their supervisors required appropriate training and supportive supervision for community sensitisation, awareness and risk communication during pandemic outbreaks, and the lack of these was noted as a significant barrier to effective service delivery. $^{79} 1316-18202125293234373840$

The nature, content and modality of training during pandemic varied immensely depending on context and specific role carved out for CHWs: as such what constitutes appropriate training for CHW might be very context specific. Components of training programmes reported focused on:

- Changes in protocols for existing service delivery pertaining to screening or managements.

- Education and awareness on the pandemic disease condition for CHWs.

- For generating awareness, risk communication and community sensitisation strategies on the pandemic condition to key client groups of CHWs or as in the area they served completely. 
- Disease surveillance, contact tracing and outbreak response.

- Transportation and management of suspect or confirmed cases of those affected by the pandemic disease condition.

- Use of personal protective equipment (PPE) and other infection control practices.

- Rebuilding community relationships postoutbreak.

In most of these studies, it was reported that appropriate training in skills and knowledge related to disease outbreaks led to an improvement in awareness of the disease, screening and reporting. In qualitative studies, it was reported that training changed in perceptions of CHWs, leading to more proactive involvement in disease prevention and control. In Sierra Leone, for instance, the Mobile Training and Support programme (an e-health training for CHWs on pandemics) used a decentralised reporting structure with outreach to individual CHWs and was reported to be useful. ${ }^{29}$ Appropriate training helped CHWs overcome fear and become more confident about providing and delivering care and helped them deal with a broad range of challenges. Transparency in communication that included sharing of information from supervisors motivated CHWs to be involved and mitigated the fear frequently encountered during pandemics.

CHWs were reported to be at an increased risk of exposure due to lack of or insufficient or incorrect usage of PPE. ${ }^{10} 181940$ In previous disease outbreaks, training sessions on pandemic preparedness were focused on protocols, and the use of appropriate and adequate PPE, during patient care. There is some evidence that training and availability of PPE resulted in CHWs being more confident to cope with managing the disease outbreak. ${ }^{10131617204042}$

During previous pandemics, CHWs have faced stigmatisation, isolation and in some cases were even socially ostracised. ${ }^{1533} 37$ The studies reported that communities often saw them as 'carriers for infection' owing to the proximity (real or perceived) with those affected by the outbreaks as well as high mortality rates among healthcare workers in general. CHWs often isolated themselves to protect their 'loved ones' from disease and stigma. As noted in the subsequent section, availability of PPE boosted confidence in CHWs but its effect on community perceptions about CHWs has not been studied.

CHWs used several coping strategies including peer support, finding renewed purpose in continuing to serve the community and turning to religion to guide strength. ${ }^{15}$ None of the studies reported preparedness to mitigate well-being for CHWS, but post facto efforts like peer and family support (praying together before work or through social media platform like WhatsApp) were reported to enhance CHW well-being. ${ }^{15}$ Furtherore, workshops that provided emotional support and ways to deal with the social stigma helped CHWs cope with seeing patients and colleagues dying from the disease. Studies reported that improved working conditions, provision of housing allowance, equal training opportunities, transportation/ remote area allowance (or provision of bicycles in rural areas), and improved salaries (paid on time and for a broad range of services), awards in high-profile public events contributed to better recruitment and retention of CHWs during pandemics. ${ }^{13} 15163334$ Some studies also noted that CHWs often had to bear the cost on their own or even borrow money to provide services or medicines to those they served-acting as an important challenge for motivation. ${ }^{1617}$

Support from community-based groups for community access and community-centred care

Community engagement and sensitisation were reported to address knowledge gaps related to disease outbreak and discourage discrimination and stigmatisation towards CHWs. ${ }^{15} 33$ Coercive laws against community members initiating discrimination and stigmatisation towards CHWs were not reported. Information sharing in the community as well as engagement of community leaders were reported to help build trust in CHWs, leading to better access as well as satisfactory experience in care received. ${ }^{88} 333537$ Community elders and village heads should be engaged and involved to rebuild trust, leading to an increased use of services. We did not find any evidence pertaining to aspects of community support and access from high-income countries.

\section{Community-level outputs and health outcomes of CHW-led pandemic response}

A study noted that the social standing (in terms of social status) of CHWs empowered them in terms of their participation in pandemic responses. ${ }^{34}$ Community health policies for social mobilisation and community engagement strategies were found to build trust and increase utilisation of services, which were found to be useful in pandemic response. ${ }^{81533} 37$ Engaging CHWs in the early phases of outbreaks potentially improved overall response procedures and adaptive resilience. ${ }^{34}$ It was reported that CHW-led programmes led to improved knowledge and awareness among farmers leading to improved motivation for reporting of avian influenza. ${ }^{7}$ In another study, it was noted that reporting for malaria by CHWs improved from $59 \%$ (preoutbreak) to $95 \%$ (during outbreak) and 98\% (postoutbreak) owing to 'sustained investments in CHWs through provision of incentives, supervision and provision of adequate reporting tools'. ${ }^{22}$ In general, training of CHWs led to improved knowledge, service quality and well-being, as has been noted in preceding sections. One study from rural Uganda reported that CHW-delivered psychosocial support to build community trust and confidence was often not available for disadvantaged and poor families owing to lack of accountability measures. ${ }^{12}$ As such, it is important to build adequate governance and reporting structures and use an equityfocused approach in programming. 
Equity, gender, accountability and economic evaluations

We did not find any economic evaluations related to CHWs in pandemic responses. Issues around equity, gender and accountability were seldom studied, including in relation to the populations to whom CHWs provided their services. Specific equity-focused studies were scarce, but female CHWs had a different set of challenges, which has been discussed in an earlier section pertaining to CHW support and well-being.

\section{Summary of inventory of resources to guide resource development}

We created three inventories to guide and expedite resource development. The first inventory (online supplementary appendix 5) compiled 24 documents. Apart from guidance documents useful for health workers (not just CHWs) during pandemics, the inventory also included resources for ensuring occupational safety and well-being and listing actions for local health departments and subnational planning.

The second inventory included 10 guidelines (online supplementary appendix 6) and advisories on selfisolation practices that have been released by various government health departments to control COVID-19. They provide instructions for the 14-day home quarantine in non-healthcare settings, that is, at home for those with close contact to COVID-19 cases, inbound travellers from affected areas and people sharing the same home with a suspect or positive COVID-19 person.

The third inventory consisted of list of 16 different IEC resources (online supplementary appendix 7). They addressed frequently asked questions, list COVID-19 myths and facts, dos and don'ts for the general public and specific communication messages for high-risk population and individuals showing symptoms available at the time of the request.

\section{DISCUSSION}

Summary of main results and their implications for policy and practice

Role of CHWS during a pandemic

Evidence from our RES pertains mostly to well-established government-run CHW programmes in LMICs. The implications of introducing new CHW programmes in the midst of a pandemic, as is being planned by the $\mathrm{UK}^{44}$ are not well understood, but considerable challenges might be expected considering the larger body of literature around community trust and systems integration being critical for success. ${ }^{45-48}$

In countries with well-established CHW programmes, roles and tasks of CHWS change substantially during pandemics. There is a need to change the normal standards of procedures of conducting routine activitiesas for example instituting 'no-touch policy'. Relevant, tailored and continuously updated guidance, training and supportive supervision should be planned for. Most common additional activities for CHW during pandemics were community awareness, engagement and sensitisation (including for countering stigma) and contact tracing. However, when CHWs were involved in contact tracing, substantial reduction in delivery of routine but essential health service delivery took place. This is crucial considering the larger body of evidence from previous pandemics showing consequences on population health owing to disruption in routine service delivery. ${ }^{43} 4950$ WHO Guidance mandates possible use of CHWs only for contact follow-up component of contact tracing. ${ }^{38}$ The bulk of the contact tracing work involves contact identification and listing, and it should be conducted by a separate trained cadre, as mandated by WHO. Effectiveness of different cadres for conducting contact tracing is beyond the scope of the RES. Yet, our evidence suggests that not involving CHWs in contact tracing may also safeguard essential primary healthcare service delivery, which is a key concern in any pandemic.

Key considerations for effectively mobilising $\mathrm{CHW}$ in a pandemic

There is a wide variation in CHW programmes in most countries in alignment with how they are defined and what they tasks they are allocated in normal times and in terms of their level of education and training, modality and degree of engagement with health system and remunerations they receive for the same. ${ }^{51}$ As such, in terms of practical decision making, the background context will determine considerations for effective CHW mobilisation during a pandemic. However, clear guidance on changed roles and tasks-vis-à-vis essential activities that need to be sustained (with modification), non-essential tasks that can be postponed and additional pandemic activities that need to be performed-is critical. As noted in our RES, disruption in supply chain, logistics and supportive supervision is expected and should be accounted for when guidance and training are being instituted. These apart, supportive structures related to governance, sustained financing and health information systems need to be carefully planned with built in strategies for course correction and updating in light of new information and developments. All these imply the need for training to be carefully planned and anticipating potential scenarios and consequent mitigation plans.

During previous pandemics, CHWs experienced stigmatisation, isolation and were socially ostracised, and the same might be expected during COVID-19 without appropriate investment and support the same. Governments should thus make provisions for psychosocial support in the form of peer support and professional help. While the RES does not provide any particular information pertaining to proactive approach to prevent stigmatisation of CHWs in the first place, general principles of antistigma programmes ${ }^{52}$ indicated community awareness and engagement can be key. As such engagement for and by CHWs with community leaders to develop culturally appropriate messages might serve the dual purpose of awareness for prevention as well as countering stigma. Futhermore, developing an a priori 
pandemic communication plan and engaging with CHWs and community leaders that aim to build trust, engage with affected populations, and integrate risk communication into health and emergency response systems would be useful.$^{53}$ Our RES indicated that training and aavailability of PPE resulted in CHWs being more confident to cope with managing the disease outbreak. The effect of PPE availability for CHWs on communities is not reported. However, an effective health system which takes care of local CHWs might potentially build community trust in addition to ensuring CHW safety .

CHWs in most contexts, and as we found in most of the included studies, have a volunteer status without any base salary, long-term security or other employment benefits like retirement benefits, medical or life insurance. ${ }^{54}$ Performance-based incentives (financial or in-kind) leads to CHWs focusing more on tasks that are remunerated. As such, in pandemic, wherein many of the ordinarily remunerated, tasks are suspended and additional tasks are being allocated might be unsettling for CHWs. More broadly, recognition as staff and moving towards a base salary structure in alignment with the 2018 WHO recommendation against a performance-based incentive structure $^{55}$ during a pandemic should be planned. This will not just be beneficial for health systems performance but is also imperative from a rights perspective. There is, however, need for more research on this domain. Provisions for additional transport allowance, accommodation and child support should also be considered owing to the need to protect families of CHWs from infection. Awards and recognition in a high-profile manner might also be useful.

In many countries most, if not all, CHWs are women and are subject to gender norms (domestic work being one example) as well as other gendered vulnerabilities and risks (like domestic violence) ${ }^{56-58}$ Sustained investment together with governance and information systems support for this cadre are important. Furthermore, as argued elsewhere, CHWs are part of a larger ecosystem of health system wherein ensuring trust and accountability is essential, more so during a pandemic. ${ }^{49}$

\section{Implications for research}

We took a broad scoping approach in the RES to understand issues around CHWs in pandemic response. Conduct of research related to health workers during pandemics is extremely challenging and, as such, highquality evidence from trials may be neither feasible nor ethical. However, the use of cross-sectional surveys, beforeand-after studies, time-series studies and qualitative study designs is feasible. Implementation research related to key pandemic response functions like contact tracing, community awareness and antistigma programmes is crucial to understand issues related to human resources for health and better pandemic control. Equity, gender and accountability dimensions together with economic evaluations are major gap in the literature that need to be studied.

\section{Strength and limitations of the review process}

In terms of the strengths of this review, a robust and transparent search strategy was used to identify relevant studies of interest. Broadening the scope to include multiple recent pandemics enabled us to get more meaningful evidence from a broader but relevant literature to inform decision making. We searched only one database, but we had extensively used other methods to identify other research. The identification of seven studies through hand-searching of websites and reference screening demonstrate the robust application and utility of the approach. We have not hand-searched government, ministry and agency websites in China and other South American countries (which have large-scale CHW programmes), but we found reviews that included studies from South American Countries. Being an RES, we acknowledge such limitations owing to the time constraints we faced. CHWs programmes are in essence a complex health systems intervention. Our broad scoping approach implies specific issues like accountability, scaling up, impact of training, education, socioeconomic conditions, supervision and magnitude of remuneration of CHWs on service delivery or effectiveness of alternatives to CHW programmes is not within the scope of the RES.

\section{Policy impact and stakeholder engagement}

We submitted the key policy considerations along with a shorter version of the RES to the requester and released it publicly on our institutional website considering the immediacy of decision making. The RES has been used nationally for the development of training resources, recommendation adoption in Odisha (a state in eastern India) and has been referred to in WHO policy note on violence against women ${ }^{56}$ during COVID-19. The inventories have also been used extensively for preparation of checklists for rural and urban primary health centre preparedness to tackle COVID-19. ${ }^{5960}$ Our detailed experience in relation to stakeholder engagement for uptake of RES will be captured in a separate paper.

\section{CONCLUSION}

In conclusion and based on our findings, CHWs play an important role in the prevention and control of pandemics like COVID-19. This is relevant and feasible in countries that already have existing CHW programmes. However, there is a need for role clarity, further training and regulation to ensure preparedness of CHWs in pandemic-like situations. The use of CHWs in countries without pre-existing CHWs programmes may be challenging considering the health systems reorientation needs and the lack of established community relationships. Applicability of available evidence on CHWs for pandemic response should be considered by such countries before embarking on ambitious CHW programmes amid a public health crisis.

Twitter Soumyadeep Bhaumik @DrSoumyadeepB and Jyoti Tyagi @drjyotityagi 
Acknowledgements Dr Rajani R Ved and Dr Neha Dumka from National Health Systems Resource Centre for inputs on scope of the rapid evidence synthesis (RES) to enhance utility in government decision making.

Contributors SB: conceptualisation, methodology, data curation, formal analysis, project administration, writing - original draft preparation, writing - review and editing. SM: conceptualisation, methodology, data curation, formal analysis, writing - original draft preparation, writing - review and editing. JT and DN: data curation, formal analysis, writing - review and editing. MK: data curation, writing - review and editing.

Funding The rapid evidence synthesis platform in India is funded by the WHO, Alliance for Health Policy and Systems Research.

Disclaimer The funder had no role in design or content of the study. Opinions expressed do not necessarily reflect the official position of the funder or the requester.

\section{Competing interests None declared.}

Patient and public involvement Patients and/or the public were not involved in the design, or conduct, or reporting, or dissemination plans of this research.

Patient consent for publication Not required.

Provenance and peer review Not commissioned; internally peer reviewed.

Data availability statement All data relevant to the study are included in the article or uploaded as supplementary information. Data made available as supplementary appendix.

Open access This is an open access article distributed in accordance with the Creative Commons Attribution 4.0 Unported (CC BY 4.0) license, which permits others to copy, redistribute, remix, transform and build upon this work for any purpose, provided the original work is properly cited, a link to the licence is given, and indication of whether changes were made. See: https://creativecommons.org/ licenses/by/4.0/.

ORCID iD

Soumyadeep Bhaumik http://orcid.org/0000-0001-9579-4453

\section{REFERENCES}

1 Iserson KV. Augmenting the disaster healthcare workforce. West $J$ Emerg Med 2020;21:490-6.

2 Adams JG, Walls RM. Supporting the health care workforce during the COVID-19 global epidemic. JAMA 2020. doi:10.1001/ jama.2020.3972. [Epub ahead of print: 12 Mar 2020].

3 Hartzler AL, Tuzzio L, Hsu C, et al. Roles and functions of community health workers in primary care. Ann Fam Med 2018;16:240-5.

4 Tricco AC, Langlois EV, Straus SE. Rapid reviews to strengthen health policy and systems: a practical guide. Geneva: World Health Organization, 2017.

5 Tricco AC, Lillie E, Zarin W, et al. PRISMA extension for scoping reviews (PRISMA-ScR): checklist and explanation. Ann Intern Med 2018;169:467-73.

6 Agarwal S, Sripad P, Johnson C, et al. A conceptual framework for measuring community health workforce performance within primary health care systems. Hum Resour Health 2019;17:86.

7 Farrell PC, Hunter C, Truong B, et al. Control of highly pathogenic avian influenza in Quang tri Province, Vietnam: voices from the human-animal interface. Rural Remote Health 2015;15:3044.

8 Gray N, Stringer B, Bark G, et al. 'When Ebola enters a home, a family, a community': a qualitative study of population perspectives on Ebola control measures in rural and urban areas of Sierra Leone. PLoS Negl Trop Dis 2018;12:e0006461.

9 Gunnlaugsson G, Hauksdóttir Íris Eva, Bygbjerg IC, et al. 'Tiny Iceland' preparing for Ebola in a globalized world. Glob Health Action 2019;12:1597451.

10 McMahon SA, Ho LS, Brown $\mathrm{H}$, et al. Healthcare providers on the frontlines: a qualitative investigation of the social and emotional impact of delivering health services during Sierra Leone's Ebola epidemic. Health Policy Plan 2016;31:1232-9.

11 ReBUILD Consortium. 'Fighting a battle': Ebola, health workers and the health system in Sierra Leone. UK: ReBUILD Research Programme Consortium, 2016

12 de Vries DH, Rwemisisi JT, Musinguzi LK, et al. The first mile: community experience of outbreak control during an Ebola outbreak in Luwero district, Uganda. BMC Public Health 2016;16:161.
13 Englert EG, Kiwanuka R, Neubauer LC. 'When I die, let me be the last.' community health worker perspectives on past Ebola and Marburg outbreaks in Uganda. Glob Public Health 2019;14:1182-92.

14 Ives J, Greenfield S, Parry JM, et al. Healthcare workers' attitudes to working during pandemic influenza: a qualitative study. BMC Public Health 2009;9:56.

15 Raven J, Baral S, Wurie $\mathrm{H}$, et al. What adaptation to research is needed following crises: a comparative, qualitative study of the health workforce in Sierra Leone and Nepal. Health Res Policy Syst 2018;16:6.

16 Wurie HR, Samai M, Witter S. Retention of health workers in rural Sierra Leone: findings from life histories. Hum Resour Health 2016;14:3.

17 Witter S, Wurie H, Chandiwana P, et al. How do health workers experience and cope with shocks? learning from four fragile and conflict-affected health systems in Uganda, Sierra Leone, Zimbabwe and Cambodia. Health Policy Plan 2017;32:iii3-13.

18 Attinsounon CA, Hounnankan CA, Dovonou CA, et al. [Knowledge and attitudes of community volunteers on Lassa and Ebola viral haemorrhagic fevers in the Donga Department (North Benin)]. Pan Afr Med J 2017;26:229.

19 Sidibé S, Camara BS, Delamou A, et al. [Knowledge, attitudes and practices of healthcare providers on suspected Ebola cases in Guinea]. Rev Epidemiol Sante Publique 2018;66:369-74.

20 Delamou A, Sidibé S, El Ayadi AM, et al. Maternal and child health services in the context of the Ebola virus disease: health care workers' knowledge, attitudes and practices in rural guinea. Afr J Reprod Health 2017;21:104-13.

21 Hemingway-Foday JJ, Ngoyi BF, Tunda C, et al. Lessons learned from reinforcing epidemiologic surveillance during the 2017 Ebola outbreak in the Likati district, Democratic Republic of the Congo. Health Secur 2020;18:S-81-S-91.

22 Vandi MA, van Griensven J, Chan AK, et al. Ebola and community health worker services in Kenema district, Sierra Leone: please mind the gap! Public Health Action 2017;7:55-61.

23 Plucinski MM, Guilavogui T, Sidikiba S, et al. Effect of the Ebolavirus-disease epidemic on malaria case management in guinea, 2014: a cross-sectional survey of health facilities. Lancet Infect Dis 2015;15:1017-23.

24 Reques L, Bolibar I, Chazelle E, et al. Evaluation of contact tracing activities during the Ebola virus disease outbreak in guinea, 2015. Int Health 2017;9:131-3.

25 Siekmans K, Sohani S, Boima T, et al. Community-Based health care is an essential component of a resilient health system: evidence from Ebola outbreak in Liberia. BMC Public Health 2017;17:84.

26 Bolkan HA, Bash-Taqi DA, Samai M, et al. Ebola and indirect effects on health service function in Sierra Leone. PLoS Curr 2014;6. doi:10.1371/currents. outbreaks.0307d588df619f9c9447f8ead5b72b2d. [Epub ahead of print: 19 Dec 2014].

27 Ameme DK, Nyarko KM, Afari EA, et al. Training Ghanaian frontline healthcare workers in public health surveillance and disease outbreak investigation and response. Pan Afr Med J 2016;25:2.

28 Dickmann P, Kitua A, Apfel F, et al. Kampala manifesto: building community-based one health approaches to disease surveillance and response-The Ebola Legacy-Lessons from a peer-led capacitybuilding initiative. PLoS Negl Trop Dis 2018;12:e0006292.

29 Mc Kenna P, Babughirana G, Amponsah M, et al. Mobile training and support (MOTS) service-using technology to increase Ebola preparedness of remotely-located community health workers (CHWs) in Sierra Leone. Mhealth 2019;5:35.

30 Otu A, Ebenso B, Okuzu O, et al. Using a mHealth tutorial application to change knowledge and attitude of frontline health workers to Ebola virus disease in Nigeria: a before-and-after study. Hum Resour Health 2016;14:5.

31 Patel U, Pharr JR, Ihesiaba C, et al. Ebola outbreak in Nigeria: increasing Ebola knowledge of volunteer health advisors. Glob J Health Sci 2015;8:72-8.

32 Stehling-Ariza T, Rosewell A, Moiba SA, et al. The impact of active surveillance and health education on an Ebola virus disease cluster - Kono District, Sierra Leone, 2014-2015. BMC Infect Dis 2016;16:611.

33 Armstrong-Mensah EA, Ndiaye SM. Global health security agenda implementation: a case for community engagement. Health Secur 2018;16:217-23.

34 Boyce MR, Katz R. Community health workers and pandemic preparedness: current and prospective roles. Front Public Health 2019;7:62.

35 Perry HB, Dhillon RS, Liu A, et al. Community health worker programmes after the 2013-2016 Ebola outbreak. Bull World Health Organ 2016;94:551-3. 
36 Elston JWT, Moosa AJ, Moses F, et al. Impact of the Ebola outbreak on health systems and population health in Sierra Leone. J Public Health 2016;38:673-8.

37 Miller NP, Milsom P, Johnson G, et al. Community health workers during the Ebola outbreak in guinea, Liberia, and Sierra Leone. J Glob Health 2018;8:020601.

38 WHO-AFRO. Contact tracing during an outbreak of Ebola virus disease Brazzaville: World Health organization African region office, 2014. Available: https://www.who.int/csr/resources/publications/ ebola/contact-tracing-during-outbreak-of-ebola.pdf [Accessed 23 Mar 2020].

39 World Health Organization. Infection prevention and control during health care when novel COVID-19 is suspected, 2020. Available: https://www.who.int/emergencies/diseases/novel-coronavirus-2019/ technical-guidance/infection-prevention-and-control

40 Selvaraj SA, Lee KE, Harrell M, et al. Infection rates and risk factors for infection among health workers during Ebola and Marburg virus outbreaks: a systematic review. J Infect Dis 2018;218:S679-89.

41 Nassar AF, Alemi F, Hetmyer A, et al. Automated monitoring to detect H1N1 symptoms among urban, Medicaid-eligible, pregnant women: a community-partnered randomized controlled trial. J Community Health 2014;39:159-66.

42 Bower H, Grass JE, Veltus E, et al. Delivery of an Ebola virus-positive stillborn infant in a rural community health center, Sierra Leone, 2015. Am J Trop Med Hyg 2016;94:417-9.

43 Elston JWT, Cartwright C, Ndumbi $P$, et al. The health impact of the 2014-15 Ebola outbreak. Public Health 2017;143:60-70.

44 Haines A, de Barros EF, Berlin A, et al. National UK programme of community health workers for COVID-19 response. Lancet 2020;395:1173-5.

45 Scott K, George AS, Ved RR. Taking stock of 10 years of published research on the ASHA programme: examining India's national community health worker programme from a health systems perspective. Health Res Policy Syst 2019;17:29.

46 Jaskiewicz W, Tulenko K. Increasing community health worker productivity and effectiveness: a review of the influence of the work environment. Hum Resour Health 2012;10:38.

47 Mishra A. 'Trust and teamwork matter': community health workers experiences in integrated service delivery in India. Glob Public Health 2014;9:960-74.

48 Pinto RM, da Silva SB, Soriano R. Community health workers in Brazil's unified health system: a framework of their praxis and contributions to patient health behaviors. Soc Sci Med 2012;74:940-7.

49 Schaaf M, Fox J, Topp SM, et al. Community health workers and accountability: reflections from an international "think-in". Int $J$ Equity Health 2018;17:66.
50 Quaglio G, Tognon F, Finos L, et al. Impact of Ebola outbreak on reproductive health services in a rural district of Sierra Leone: a prospective observational study. BMJ Open 2019;9:e029093.

$51 \mathrm{WHO}$. Who guideline on health policy and system support to optimize community health worker programmes Geneva: World Health organization 2018. Available: https://apps.who.int/iris/ bitstream/handle/10665/275474/9789241550369-eng.pdf

52 Yanos PT, Lucksted A, Drapalski AL, et al. Interventions targeting mental health self-stigma: a review and comparison. Psychiatr Rehabil J 2015;38:171-8.

53 WHO. Communicating Risk in Public Health Emergencies : A WHO Guideline for Emergency Risk Communication (ERC) policy and practice Geneva: World Health Organisation 2017. Available: https:// apps.who.int/iris/bitstream/handle/10665/259807/9789241550208eng.pdf;jsessionid=5C8DB861A3900CA529331F4C724C3218? sequence $=2$

54 Scott K, Beckham SW, Gross M, et al. What do we know about community-based health worker programs? A systematic review of existing reviews on community health workers. Hum Resour Health 2018;16:39.

55 Cometto G, Ford N, Pfaffman-Zambruni J, et al. Health policy and system support to optimise community health worker programmes: an abridged who guideline. Lancet Glob Health 2018;6:e1397-404.

56 WHO. COVID-19 and violence against women : What the health sector/system can do: World Health Organisation, 2020. Available: https://apps.who.int/iris/bitstream/handle/10665/331699/WHOSRH-20.04-eng.pdf?ua=1 [Accessed 27 Apr 2020].

57 Sarin E, Lunsford SS. How female community health workers navigate work challenges and why there are still gaps in their performance: a look at female community health workers in maternal and child health in two Indian districts through a reciprocal determinism framework. Hum Resour Health 2017;15:44.

58 George A. Human resources for health: a gender analysis women and gender equity knowledge network and the health systems knowledge network of the who Commission on social determinants of health, 2007

59 COVID-19-PHC Action Group. COVID-19 preparedness checklist for rural primary health care and community settings. Available: https:// www.georgeinstitute.org.in/covid-19-preparedness-checklist-forrural-primary-health-care-community-settings [Accessed $27 \mathrm{Apr}$ 2020].

60 COVID-19-PHC Action Group. COVID-19 preparedness checklist for urban primary health centres in India. Bengaluru: Insitute of Public Health, 2020. https://iphindia.org/wp-content/uploads/2020/ 04/COVID-19-Preparedness-Checklist-for-Urban-Primary-HealthCentres-in-India-.pdf 